\title{
Efeitos de diferentes intensidades de exercício sobre o gasto energético e a sensação de fome em jovens
}

\author{
Effects of different exercise intensities on energy expenditure \\ and hunger sensation in young adults
}

\author{
Tatiana Acioli Lins \\ Pedro Rogério da Silva Neves \\ Manoel da Cunha Costa \\ Wagner Luiz do Prado
}

1 Universidade de Pernambuco. Escola Superior de Educação Física. Recife, PE. Brasil.

Submetido em 18/09/09 Revisado em 13/02/10 Aprovado em 18/04/10
Resumo - O presente estudo objetiva investigar os efeitos agudos e de curto prazo ( 2 horas) de diferentes intensidades de exercício aeróbio sobre o gasto energético pós-exercício, a razão de troca respiratória (QR) e a sensação de fome em homens. Participaram do estudo, 20 sujeitos eutróficos $\left(22,41 \pm 1,76 \mathrm{~kg} / \mathrm{m}^{2}\right)$ e praticantes de exercício físico. Todos os sujeitos foram submetidos, aleatoriamente, a quatro condições experimentais: controle (sem exercício); EBI, exercício de baixa intensidade $\left(40 \%\right.$ do $\left.\mathrm{VO}_{2 \text { pico }}\right)$; EMI, exercício de moderada intensidade $\left(60 \%\right.$ do $\left.\mathrm{VO}_{2 \text { pico }}\right)$ e EAI, exercício de alta intensidade $\left(80 \%\right.$ do $\left.\mathrm{VO}_{2 \text { pico }}\right)$. A magnitude do (EPOC - consumo excessivo de oxigênio após o exercício) e o comportamento do QR foram mensurados através de análise direta de gases. A escala visual analógica foi utilizada para avaliar a sensação de fome em (basal, imediatamente após a sessão experimental, aos 30, 60, 90 e 120 minutos de recuperação passiva). Os resultados demonstraram que o EPOC teve uma correlação positiva com a intensidade do exercício $(r=0,74, p<0,01)$, sendo a duração e a magnitude dependentes da intensidade do exercício. Entre todas as condições experimentais, a sensação de fome ao término do exercício foi maior no EBI ( $p<0,01)$. Ao longo do período de 120 minutos de recuperação passiva, a sensação de fome aumentou independente da condição experimental. A partir dos resultados, podemos sugerir que após o EAI a magnitude do EPOC é maior, promovendo maior gasto energético no pós-exercício, com um concomitante aumento na utilização de gordura (menor QR) e supressão transitória da sensação de fome em adultos jovens.

Palavras-chave: Consumo de oxigênio; Metabolismo; Fome; Ingestão Alimentar.

Abstract - The aim of this study was to investigate the acute and short-term effects of different aerobic exercise intensities on postexercise energy expenditure, respiratory exchange ratio (RER), and hunger sensation in young adults. Twenty healthy, physically active ( $>6$ months) young men of normal weight $\left(22.41 \pm 1.76 \mathrm{~kg} / \mathrm{m}^{2}\right)$ participated in this study. All subjects were randomly submitted to four experimental conditions: control (no exercise); LIE, low-intensity exercise ( $40 \%$ of $\left.\mathrm{VO}_{\text {2peak }}\right)$; $\mathrm{MIE}$, exercise of moderate intensity $\left(60 \%\right.$ of $\left.\mathrm{VO}_{2 \text { peak }}\right)$, and $\mathrm{HIE}$, high-intensity exercise $\left(80 \%\right.$ of $\left.\mathrm{VO}_{2 \text { peak }}\right)$. The magnitude of (EPOC - excess post-exercise oxygen consumption), and RER were measured by direct gas analysis. A visual analogue scale was used to rate hunger sensation at baseline, immediately after the experimental session, and after 30, 60, 90 and 120 minutes of passive recovery. The results showed a positive correlation between the magnitude of EPOC and exercise intensity $(r=0.74, p<0.01)$, with the duration and magnitude depending on exercise intensity. For all experimental conditions, the hunger sensation was greater in the LIE group $(p<0.01)$. The hunger scores increased over the 120 minutes of passive recovery in all exercise and control sessions. The results suggest that the magnitude of EPOC is higher after HIE, promoting higher postexercise energy expenditure and a concomitant increase in fat utilization (lower RER) and transient suppression of hunger sensation in young adults.

Key words: Oxygen Consumption; Metabolism; Hunger; Food intake. 


\section{INTRODUÇÃO}

Estimativas sugerem que, por ano, 40\% das mulheres americanas são submetidas a terapias para redução e/ou controle da massa corporal, utilizando, na maioria das vezes, o exercício físico como uma das estratégias ${ }^{1}$; entretanto, a capacidade do exercício de induzir um estado de balanço energético negativo depende não apenas do gasto energético advindo do esforço, mais também da ingestão alimentar pós-exercício.

Nos últimos anos, o exercício físico foi reconhecido como um potente regulador da fome, apetite, balanço energético (BE) e da massa corporal². Pomerleau et al. ${ }^{3}$ sugerem que a prática de exercícios físicos colabora apenas de forma sutil com a redução da massa corporal, devido a uma possível compensação na ingestão alimentar e diminuição nas atividades físicas espontâneas.

Vários estudos têm verificado os efeitos de diferentes tipos e intensidades de exercício físico sobre a ingestão alimentar ${ }^{4,6,7}$. Alguns autores reportam aumento na ingestão energética nos períodos subsequentes ao esforço ${ }^{5}$, outros estudos verificaram não haver efeitos do exercício sobre o apetite ${ }^{6,7} \mathrm{e}$ ingestão alimentar ${ }^{1,33}$, e outros relatam supressão do apetite $^{2,4,6}$ e diminuição na ingestão alimentar ${ }^{6,30}$. Entretanto, é importante ressaltar que a supressão da ingestão alimentar pós-exercício, conhecida como anorexia induzida pelo exercício, é de curta duração, sendo verificado um aumento da fome após 15 minutos de recuperação ${ }^{6}$.

Além do gasto energético promovido pelo exercício em si e da ingestão energética, o consumo excessivo de oxigênio pós-exercício (EPOC) também é um fator essencial na determinação do balanço energético, visto que é um indicador do gasto energético pós-exercício. Está bem estabelecido que, após o término do exercício, o consumo de oxigênio não retorna aos valores de repouso imediatamente (EPOC) ${ }^{8}$.

Os resultados sobre magnitude e duração do EPOC, bem como sua real contribuição para indução do balanço energético negativo ainda são controversos. Vários estudos demonstram que o EPOC pode permanecer por horas ${ }^{10,11}$, outros propõem que seu efeito é de curta duração ${ }^{12,13}$; entretanto, é consenso na literatura que o tempo e a magnitude do EPOC são determinados pela duração, intensidade, utilização de substratos e tipo de exercício ${ }^{12,14}$, bem como a ingestão alimentar.

Essas divergências nos resultados se devem, provavelmente, a diferenças metodológicas, como o tipo, duração e a intensidade do exercício, o estado nutricional dos indivíduos, composição da refeição pré-teste, e o tempo decorrido entre o exercício e a ingestão alimentar ${ }^{4}$.

Por isso, faz-se necessário conhecer os efeitos de diferentes intensidades de exercício aeróbio sobre a sensação de fome, apetite e o gasto energético pós-exercício, para que o exercício físico seja utilizado como uma ferramenta para prevenção e/ou tratamento de distúrbios relacionados ao balanço energético.

Assim, o presente estudo objetiva investigar os efeitos agudos e de curto prazo de diferentes intensidades de exercício aeróbio sobre o gasto energético pós-exercício, a razão de troca respiratória $(\mathrm{QR})$ e a sensação de fome em homens.

\section{PROCEDIMENTOS METODOLÓGICOS}

\section{Amostra}

Participaram deste estudo, 20 homens $(21,2 \pm 2,58$ anos) saudáveis, universitários, que se voluntariaram, após leitura de cartazes/convite distribuídos pelo campus. Os critérios de inclusão foram: ser praticante de exercício físico ( $\geq 6$ meses, 3 vezes/ semana) e eutróficos (IMC $=22,41 \pm 1,76 \mathrm{~kg} / \mathrm{m}^{2}$ ). Foram estabelecidos como critérios de exclusão: consumo crônico de bebida alcoólica, fumo ou uso de suplementos alimentares, uso de produtos farmacológicos, e terem apresentado variação na massa corporal $( \pm 2 \mathrm{~kg})$ nos últimos seis meses. O estudo foi aprovado pelo Comitê de Ética da Universidade de Pernambuco (154/08). Todos os voluntários assinaram o termo de consentimento livre e esclarecido.

\section{Desenho experimental}

Este estudo caracteriza-se como crossover, no qual todos os voluntários foram submetidos, de forma aleatória (sorteio), a todas as condições experimentais, sendo eles controles deles mesmos. Os sujeitos realizaram cinco visitas ao laboratório. Durante a primeira visita ao laboratório, foram coletadas as medidas antropométricas, composição corporal e consumo de oxigênio $\left(\mathrm{VO}_{2}\right)$. Da segunda até a quinta visita, os sujeitos chegaram ao laboratório às 7:00h, após jejum noturno de 12h. Nesse momento, consumiam um lanche padrão com conteúdo energético de 350 kcal (Carboidrato: 61,7\%; Proteína: 13,4\% e Lipídeos: 24,9\%). Trinta minutos após a ingestão do lanche ( $\approx$ às 7:30h), os sujeitos foram submetidos, de forma aleatória e com intervalo de sete dias (wash out) entre cada sessão, a quatro 
condições experimentais: 1) Controle: os sujeitos eram mantidos em repouso (sentados - era apenas permitido ler e/ou utilizar o computador); 2) Exercício de baixa intensidade (EBI): os sujeitos se exercitaram em esteira rolante na intensidade de esforço correspondente a $40 \%$ do $\mathrm{VO}_{2 \text { pico }}$; 3) Exercício de moderada intensidade (EMI): os sujeitos se exercitaram na intensidade de esforço correspondente a $60 \%$ do $\mathrm{VO}_{\text {2pico }}$ e 4) Exercício de alta intensidade (EAI): os sujeitos se exercitaram na intensidade de esforço correspondente a $80 \%$ do $\mathrm{VO}_{\text {2pico }}$.

Todas as sessões experimentais foram conduzidas no mesmo horário do dia, para evitar quaisquer variações circadianas. Todos os procedimentos foram conduzidos no laboratório com temperatura constante $\left(24^{\circ} \pm 1 \mathrm{C}\right)$. Os sujeitos foram orientados a não realizar nenhum tipo de exercício físico, por um período de $48 \mathrm{~h}$ antes de cada sessão.

As sessões de exercício físico foram isocalóricas, com gasto energético de $350 \mathrm{kcal}$ (o que variava entre cada sessão experimental era o tempo de exercício). Durante todo período de exercício, foi realizada análise direta de gases expirados. Após o término de cada sessão de exercício ou na sessão controle, os sujeitos permaneciam sentados (recuperação passiva) por 120 minutos, para determinação do gasto energético.

A sensação de fome foi mensurada em seis momentos distintos: antes, imediatamente após cada sessão experimental, e aos 30, 60, 90 e 120 minutos de recuperação passiva.

\section{Medidas antropométricas e composição corporal}

A massa corporal foi obtida através de balança com escala de precisão $0,1 \mathrm{~kg}$ (Fillizola) e a estatura através de estadiômetro de madeira, seguindo a metodologia proposta por Jackson $\&$ Pollock ${ }^{15}$ Posteriormente, foi calculado o índice de massa corpórea (IMC). A composição corporal foi predita através de impedância bioelétrica, utilizando-se o equipamento da marca Biodynamics, modelo A-310 body composition analyzing, seguindo-se os critérios descritos pelo manual do equipamento e demais procedimentos estabelecidos na literatura $^{16}$.

Consumo pico de oxigênio $\left(\mathrm{VO}_{2 \text { pico }}\right)$

$\mathrm{O}$ consumo pico de oxigênio $\left(\mathrm{VO}_{2 \text { pico }}^{2 \text { pico }}\right)$ foi mensurado através de análise direta de gases, em teste incremental em esteira rolante, utilizando-se o protocolo proposto por McConnel ${ }^{17}$. Os critérios estabelecidos para interrupção do teste foram: exaustão voluntária, coeficiente respiratório acima de 1.15, e Escala de Borg acima de 18. Uma vez que nenhum dos sujeitos atingiu o platô de consumo de oxigênio, o maior valor de $\mathrm{VO}_{2}$ encontrado antes da interrupção do teste foi adotado como sendo $\mathrm{VO}_{\text {2pico }}$ do voluntário.

O consumo de oxigênio e a produção de dióxido de carbono foram obtidos por um analisador metabólico de circuito aberto (Córtex Biophysik Metalyzer IIB) com leitura a cada 15 segundos, com utilização de máscara (Hans Rudolph). O sistema foi calibrado para volume (seringa de calibração de volume - 3L Cardioequipo), pressão atmosférica (Barômetro- Barigo) e mistura de gases (White Martins- $\left[\mathrm{O}_{2=} 12,2\right.$ e $\left.\mathrm{CO}_{2=} 4,8\right]$ ) em cada teste realizado.

$\mathrm{O} \mathrm{VO}_{2}$ e o $\mathrm{VCO}_{2}$ foram avaliados durante todo o período experimental. Para determinação do consumo de oxigênio pós-exercício (EPOC), foi realizada uma nova análise direta de gases, por dez minutos, nos intervalos de tempo (30, 60, 90 e 120 minutos de recuperação) até que se completasse o tempo total de recuperação passiva de 120 minutos. A magnitude do EPOC foi obtida a partir da soma dos valores obtidos.

\section{Gasto energético}

O gasto energético durante e pós-exercício foi determinado através de calorimetria indireta, a partir do $\mathrm{VO}_{2}$ e $\mathrm{VCO}_{2}$ obtidos na análise direta de gases. A razão de oxidação de carboidratos e de gorduras foi calculada a partir do $\mathrm{VO}_{2}$ e o do quociente respiratório (QR), baseando-se na suposição de que a contribuição das proteínas para este tipo de esforço é desprezível durante o exercício ${ }^{18}$.

\section{Sensação de fome}

A sensação de fome foi obtida através de uma escala analógica visual (EAV), em seis momentos distintos: antes, imediatamente após a sessão, aos 30, 60, 90 e 120 minutos de recuperação passiva. A EAV consiste em uma linha horizontal de 100 milímetros, que retrata o máximo da sensação de fome que o indivíduo possa apresentar na extremidade direita e na extremidade esquerda, pouca ou nenhuma sensação de fome ${ }^{19}$. Os voluntários foram orientados a marcar uma linha vertical no ponto em que a sua sensação de fome se aproximasse da extremidade referida, para mais ou para menos na sua sensação de fome. $O$ ponto marcado pelo indivíduo foi então medido com auxílio de uma régua, da extremidade esquerda (pontuação mínima 0 $\mathrm{mm}$ ) para direita, (pontuação máxima $100 \mathrm{~mm}$ ) para determinação dos escores de fome. 
A escala analógica visual apresenta alta capacidade de predizer a ingestão alimentar em indivíduos adultos jovens, e a alta reprodutibilidade tem sido demonstrada em vários estudos ${ }^{19,20}$.

\section{Análise estatística}

Todos os procedimentos estatísticos foram realizados através do Statistica versão 6.0. O teste de normalidade utilizado foi o de Shapiro-Wilk. ANOVA One-Way para medidas repetidas foi utilizada para analisar os efeitos das sessões experimentais (EBI, EMI, EAI e Controle) no gasto energético pósexercício e para diferenças intrassessão. ANOVA Two-Way para medidas repetidas foi utilizada para avaliar os efeitos das intervenções (Controle, EBI, EMI e EAI) e do tempo (antes, imediatamente após, e aos 30, 60, 90 e 120 minutos de recuperação passiva), na sensação de fome e no QR, com o post-hoc Tukey. Os valores estão apresentados em média \pm desvio padrão. $O$ nível de significância foi fixado em $\mathrm{p} \leq 0,05$.

\section{RESULTADOS}

Inicialmente, 26 indivíduos se voluntariaram para participar da pesquisa. Entretanto, seis voluntários não completaram todos os procedimentos experimentais e foram excluídos da amostra. A tabela 1 apresenta as características antropométricas, composição corporal e $\mathrm{VO}_{\text {2pico }}$ dos 20 voluntários que completaram todos os procedimentos experimentais.

Uma vez que as sessões de exercício foram isocalóricas $(\approx 350 \mathrm{kcal})$, o tempo total da sessão de exercício diferiu, significantemente $(p<0,05)$, entre as intensidades ( $\mathrm{EBI}=65,3 \pm 4,9$ minutos; $\mathrm{EMI}=45,3 \pm 5,2$ minutos; $\mathrm{EAI}=29,5 \pm 3,7$ minutos). Todas as sessões de exercício promoveram uma maior utilização tanto de carboidratos quanto de gorduras, quando comparadas à sessão controle $(\mathrm{p}<0,05)$. Houve um maior consumo de carboidratos no EMI e EAI do que no EBI $(p<0,01)$. A sessão EBI apresentou maior consumo de lipídeos em relação às demais $(\mathrm{p}<0,01)$ (Tabela 2$)$.

Tabela 1. Características antropométricas, composição corporal e $\mathrm{VO}_{2 \text { pico }}$ dos voluntários.

\begin{tabular}{lc}
\hline Variáveis & Média \pm DP \\
\hline Idade (anos) & $21,2 \pm 2,6$ \\
Massa corporal $(\mathrm{kg})$ & $70,3 \pm 10,3$ \\
Massa gorda $(\%)$ & $15,5 \pm 3,4$ \\
Estatura $(\mathrm{cm})$ & $176,7 \pm 7,4$ \\
$\mathrm{IMC}\left(\mathrm{kg} / \mathrm{m}^{2}\right)$ & $22,4 \pm 1,8$ \\
$\mathrm{VO}_{2 \text { pico }}\left(\mathrm{ml}^{2} \mathrm{~min}{ }^{1} \cdot \mathrm{kg}^{-1}\right)$ & $50,3 \pm 4,7$ \\
$\mathrm{VO}_{2 \text { pico }}\left(\mathrm{L} / \mathrm{min}^{\mathrm{m}}\right)$ & $3,5 \pm 0,4$ \\
\hline
\end{tabular}

$\mathrm{IMC}=$ índice de massa corporal; $\mathrm{VO}_{2 \text { pico }}=$ consumo pico de oxigênio.

Observou-se, nesse estudo, que após 2 horas de recuperação passiva, o gasto energético (GE) foi maior $(\mathrm{p}<0,05)$ no EAI $(157,1 \pm 14,4 \mathrm{Kcal})$ do que nas demais condições experimentais; no entanto, ao se verificar o metabolismo glicídico não houve nenhum efeito dependente da intensidade, sendo o consumo de carboidratos após o exercício sem diferenças entre as intensidades $(\mathrm{p}<0,01)$. Foi observado que quanto

Tabela 2. Efeitos de diferentes intensidades de exercício sobre o gasto energético, o EPOC e o QR

\begin{tabular}{lcccc}
\hline & Controle & EBI & EMI & EAI \\
\hline Tempo (minutos) & 30 & $65,31 \pm 4,95$ & $45,26 \pm 5,18^{*}$ & $29,55 \pm 3,70^{* \S}$ \\
GE (kcal) & $47,46 \pm 0,98$ & $352,45 \pm 0,51^{*}$ & $352,45 \pm 0,51^{*}$ & $352,45 \pm 0,51^{*}$ \\
GE CHO (kcal) & $24,70 \pm 5,76$ & $227,12 \pm 3,62^{*}$ & $258,22 \pm 15,09^{* *}$ & $273,16 \pm 22,30^{* *}$ \\
GE Lip (kcal) & $22,76 \pm 1,23$ & $125,33 \pm 0,85^{*}$ & $94,94 \pm 4,06^{* *}$ & $78,93 \pm 4,97^{* *}$ \\
GE pós-exercício (kcal) & ------- & $125,39 \pm 6,15^{*}$ & $137,28 \pm 11,70^{* *}$ & $157,14 \pm 15,40^{* * \S}$ \\
GE CHO pós-exercício (kcal) & ------ & $57,40 \pm 7,40^{*}$ & $52,69 \pm 12,05^{*}$ & $48,97 \pm 15,11^{*}$ \\
GE Lip pós-exercício (kcal) & -------- & $67,90 \pm 4,41^{*}$ & $84,59 \pm 5,10^{* *}$ & $108,17 \pm 9,23^{* * \S}$ \\
QR 30 minutos & $0,84 \pm 0,06$ & $0,83 \pm 0,05$ & $0,88 \pm 0,07^{* *}$ & $0,88 \pm 0,06^{* *}$ \\
QR 60 minutos & $0,84 \pm 0,08$ & $0,82 \pm 0,05$ & $0,86 \pm 0,06^{*}$ & $0,85 \pm 0,07$ \\
QR 90 minutos & $0,83 \pm 0,02$ & $0,81 \pm 0,02^{*}$ & $0,84 \pm 0,02^{*}$ & $0,83 \pm 0,03^{*}$ \\
QR 120 minutos & $0,83 \pm 0,02$ & $0,80 \pm 0,02^{*}$ & $0,82 \pm 0,02$ & $0,80 \pm 0,03^{* \S}$ \\
\hline
\end{tabular}

*vs controle; ¥ vs EBI; § vs EMI. ANOVA one-way, post hoc de Tukey; Diferença significativa para p<0,05. EBI= Exercício de Baixa Intensidade; $\mathrm{EMI}=$ Exercício de Moderada Intensidade= EAI, Exercício de Alta Intensidade; GE= gasto energético; GE CHO= gasto energético de carboidratos; GE Lip = gasto energético de lipídeos; QR= quociente respiratório. 
maior a intensidade do exercício maior o gasto energético advindo dos lipídeos, e o EAI (108,2×9,2 Kcal) apresentou maiores valores do que todas as demais condições experimentais. Vale ressaltar que, em todas as condições, o exercício promoveu maior utilização de gordura, quando comparado à situação controle $(22,76 \pm 5,51 \mathrm{Kcal})(\mathrm{p}<0,01)$.

Os resultados do presente estudo demonstram haver um efeito tempo dependente na redução do $\mathrm{QR}$, durante duas horas de recuperação passiva (tabela 2). Após 30 minutos no EMI e no EAI, os valores do $\mathrm{QR}$ ainda encontram-se elevados ( $<<0,05)$, em comparação ao EBI e ao controle. Após 60 minutos de recuperação passiva, apenas o EMI apresenta valores elevados do $Q R(p<0,01)$ e a partir de 90 minutos de recuperação passiva, os valores do EBI apresentam-se menores $(\mathrm{p}<0,01)$ em relação às outras condições experimentais. Ao final de 2 horas, o EBI e EAI apresentaram o QR abaixo dos valores controle $(\mathrm{p}<0,05)$.

Com relação à sensação de fome basal dos voluntários, os dados revelam não haver diferenças entre as condições experimentais (Figura 1). Imediatamente após a sessão experimental, os escores agudos de fome foram diminuídos no EMI e no EAI ( $p<0,01)$, e foram aumentados para todas as evoluções de tempo no EBI $(p<0,01)$. A sensação de fome foi maior no EBI do que nos outros grupos experimentais. Ao longo do tempo, os escores de pontuação da sensação de fome tenderam a aumentar independente da intervenção; no entanto, a supressão da fome foi maior na sessão de EAI, quando a sensação de fome começou a ser elevada 60 minutos após a sessão experimental, e após 120 minutos de recuperação passiva ainda não havia retornado aos valores basais.

\section{DISCUSSÃO}

A proposta deste estudo foi examinar os efeitos agudos e de curto prazo (120 minutos) de diferentes intensidades de exercício sobre o gasto energético pós-exercício, QR e a sensação de fome em adultos jovens. A hipótese era que o exercício de alta intensidade induziria um maior GE pós-exercício, refletindo em maior magnitude do EPOC, um menor QR (maior utilização de lipídeos), além da supressão da fome, do que o exercício de baixa e moderada intensidade.

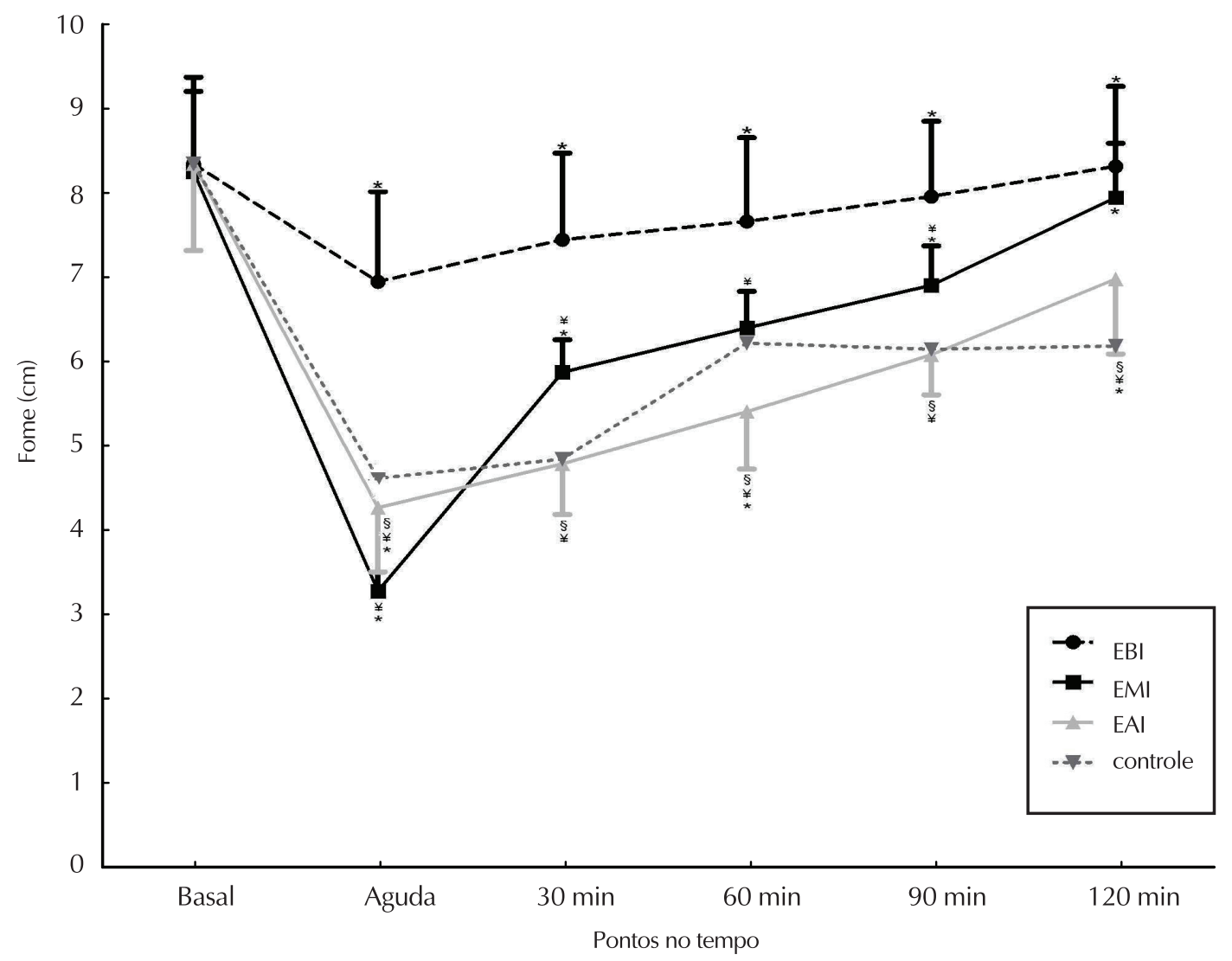

Figura 1. Efeitos de diferentes intensidades de exercício na sensação de fome.

${ }^{*}$ vs controle; ${ }^{*}$ vs EBI; ${ }^{\S}$ vs EMI. ANOVA two-way, post hoc de Tukey; Diferença significativa para p <0,05. EBI, Exercício de Baixa Intensidade; EMI, Exercício de Moderada Intensidade; EAI, Exercício de Alta Intensidade. 
Ao longo da última década, muita importância tem sido dada ao componente intensidade do exercício, como forma de facilitar a regulação do $\mathrm{GE}^{3}$. Como efeito do aumento da intensidade do exercício, há um aumento no gasto energético, promovendo um maior EPOC e oxidação de gordura ${ }^{8,10}$. $\mathrm{O}$ gasto energético pós-exercício continua estimulado por um período de tempo de 4 a $5 \mathrm{~h}$, dependendo da intensidade, da duração, e de outros fatores ${ }^{10}$.

Considerando a intensidade do exercício como determinante para o aumento no gasto energético pós-exercício, Borsheim e $\mathrm{Bahr}^{21}$ sugerem que, após atividades de alta intensidade (acima de $\left.70 \% \mathrm{VO}_{2 \max }\right)$, há um aumento linear na duração e magnitude do EPOC. Tal associação foi previamente descrita?.

Evidências ${ }^{21}$ sugerem não haver grandes alterações no EPOC após estímulos de baixa intensidade (abaixo de $50 \% \mathrm{VO}_{2 \max }$ ) e com gasto de $300 \mathrm{Kcal}$, aproximadamente, o que vem ao encontro dos nossos achados, uma vez que encontramos maior gasto energético pós-exercício após as sessões de moderada e alta intensidade, sendo maiores os valores em resposta à sessão de alta intensidade.

Uma possível resposta para o consumo elevado de $\mathrm{O}_{2}$ logo após a realização de exercício de alta intensidade é a utilização deste para restaurar a creatina fosfato no músculo esquelético, restabelecer os estoques de $\mathrm{O}_{2}$ no sangue e nos tecidos, e ativar a gliconeogênese. Outros fatores que podem influenciar o EPOC são a temperatura elevada e determinados hormônios circulantes, como adrenalina e noradrenalina ${ }^{22}$. Tais alterações são mais pronunciadas após exercícios vigorosos ou de longa duração.

Com relação ao QR pós-exercício, verifica-se no presente estudo um efeito tempo dependente na redução desta variável para todas as condições de exercício, sendo que a partir de 60 minutos de recuperação passiva, não existem diferenças entre as diferentes intensidades. A redução do QR é mais perceptível após exercícios de alta intensidade que provocam elevados índices ventilatórios e promovem extenso estresse na regulação do equilíbrio ácido-base que pode, em parte, ser explicado por uma condição transitória e ascendente representada por um tipo de hiperventilação relativa ${ }^{23}$. Entretanto, tal padrão não foi observado no presente estudo, sendo os menores valores encontrados no EBI.

O exercício possui a capacidade de desequilibrar muitas variáveis homeostáticas. O músculo esquelético produz grandes quantidades de lactato, que geram um aumento da acidez intra e extracelular, acarretando grandes aumentos na demanda de
$\mathrm{O}_{2}$ utilizado pelos músculos e grande quantidades de $\mathrm{CO}_{2}$ produzidos, modificando substancialmente $\mathrm{o} \mathrm{QR}^{24}$, e a utilização dos substratos.

Entretanto, deve-se salientar que um dos aspectos que contribui para o baixo $\mathrm{QR}$, principalmente, na recuperação de atividades intensas, pode ser o restabelecimento das reservas de bicarbonato ${ }^{23}$. Após ser utilizado de forma mais acentuada nas reações de tamponamento, em resposta a alterações na acidose do meio, o bicarbonato para ser ressintetizado necessita da incorporação de $\mathrm{CO}_{2}$ em sua estrutura molecular, resultando em um menor $\mathrm{QR}^{23,24}$. Tal mecanismo pode fornecer uma falsa ideia de aumento na oxidação de gorduras. Devido a tais dificuldades metodológicas, é muito difícil afirmarmos que o exercício de alta intensidade promove elevação do metabolismo lipídico pós-exercício ${ }^{22,25}$.

O efeito do exercício sobre a regulação da fome e da ingestão alimentar envolve, pelo menos, dois processos: um aumento no efeito anorexígeno e um concomitante aumento na eficiência saciante da refeição. A modulação desses mecanismos a partir do exercício físico pode influenciar o controle da massa corporal ${ }^{20,26}$.

Em nosso estudo, o exercício físico, independente da intensidade, foi capaz de promover redução aguda na sensação de fome. Este efeito também foi observado por outros pesquisadores ${ }^{27}$, que submeteram homens jovens a uma sessão de exercício aeróbio durante 60 minutos a $70 \%$ do $\mathrm{VO}_{2 \max }$, havendo supressão na sensação de fome imediatamente após o exercício. Outro estudo ${ }^{28}$ analisou o efeito do exercício físico $\left(72 \%\right.$ do $\left.\mathrm{VO}_{2 \max }\right)$ sobre a sensação de fome em 9 homens adultos eutróficos. Os autores observaram um estado de supressão da fome imediatamente após o exercício.

Vários estudos ${ }^{3,4}$, assim como o presente, têm demonstrado que, após uma breve supressão da fome, o exercício físico causa aumento compensatório na sensação de fome em curto prazo, sendo este um dos principais obstáculos para a indução de balanço energético negativo, com consequente redução da massa corporal.

Um possível mecanismo envolvido na anorexia induzida pelo exercício (aeróbio) é a redução das concentrações circulantes de ghrelina acilada, um potente hormônio orexígeno ${ }^{31}$. Outra hipótese estaria relacionada à termorregulação, em que altas temperaturas corporais, como as observadas em resposta ao esforço físico, promoveriam uma redução da fome/apetite ${ }^{29,30} \mathrm{e}$ ambas situações apresentam apenas efeitos agudos, alterando-se com o passar 
do tempo de recuperação.

Conforme discutido até o presente momento, parece claro na literatura que o exercício de alta intensidade leva a um estado anoréxico transitório, porém tal fenômeno não é muito bem evidenciado em resposta a atividade de moderada ou de baixa intensidade ${ }^{31}$. Uma vez que nossos achados revelam redução da fome mesmo após exercício de baixa $\left(40 \% \mathrm{VO}_{2 \text { pico }}\right)$ e moderada $\left(60 \% \mathrm{VO}_{2 \text { pico }}\right)$ intensidade, este achado pode ser um dos mais importantes do presente estudo.

\section{CONCLUSÃO}

De acordo com os resultados apresentados, podemos concluir que o exercício físico de alta intensidade promove uma elevação no gasto calórico pósexercício, com concomitante diminuição do valor do quociente respiratório pós-exercício, resultando, provavelmente, em uma maior utilização de gordura, com a indução de um estado anoréxico transitório.

Dessa forma, fica claro que, além do importante papel do exercício físico no aumento do gasto energético, é essencial que levemos em consideração, também, as alterações nos sistemas de controle da ingestão alimentar (fome, apetite e ingestão calórica), para que possamos exercer um controle mais eficiente sobre o balanço energético de indivíduos submetidos à prática regular de atividade física.

\section{Agradecimentos}

Os autores agradecem a FACEPE/CNPq e a Universidade de Pernambuco pelo suporte financeiro, a diretoria da Escola Superior de Educação Física (ESEF/UPE), as nutricionistas Rosa Idalina e Roberta Costi. Especialmente a todos os voluntários.

\section{REFERÊNCIAS BIBLIOGRÁFICAS}

1. George VA, Morganstein A. Effect of moderateintensity exercise on acute energy intake in normal and overweight females. Appetite 2003;40:43-4.

2. Hagobian T, Sharoff CG, Braun B. Effects of short-term and energy surplus on hormones related to regulation of energy balance. Metabolism 2008;57:393-8.

3. Pomerleau M, Imbeault P, Parker T, Doucet E. Effects of exercise intensity on food intake and appetite in women. Am J Clin Nutr 2004;80:1230-6.

4. Maraki M, Tsofliou F, Pitsiladis YP, Malkova D, Mutrie N, Higgins S. Acute effects of a single exercise class on appetite, energy intake and mood. Is there a time of day effect? Appetite 2005;45:272-8.

5. Verger P, Lanteaume MT, Louis-Sylvestre J. Free food choice after acute exercise in men. Appetite1994;22:159-64.
6. King NA, Burley VJ, Blundell JE. Exercise induced suppression of appetite. Effects on food intake and implications for energy balance. Eur J Clin Nutr 1994;50:663-7.

7. Imbeault P, Saint-pierre S, Almeras N, Tremblay A. Acute effects of exercise on energy intake and feeding behavior. Br J Nutr 1997;77:511-21.

8. Foureaux G, Kelerson MCP, Damaso A. Efeito do consumo excessivo de oxigênio após exercício e da taxa metabólica de repouso no gasto energético. Rev Bras Med Esporte 2006;12(6):393-8

9. Lima-Silva AE, Pires FO, Oliveira F, De-Oliveira FR, Kiss MAPDM. Metabolismo lipídico e gasto energético durante o exercício. Rev Bras Cineantropom Desempenho Hum 2008;10(3):308-12

10. Sedlock DA. Effect of exercise intensity on postexercise energy expenditure in women. Br J Sp Med 1991;25:38-40.

11. Bahr RI, Ignes I, Vaage O, Sejersted OM, Newsholme E. A. Effect of duration of exercise on excess post-exercise O2 consumption. J Appl Physiol 1992;62:485-90.

12. Maresh CM, Abraham A, De Souza, MJ. Oxygen consumption following exercise of moderate-intensity and duration. Eur J Appl Physiol 1992;65:421-6.

13. Hagberg JM, Mullin JP, Nagle FJ. Effect of work intensity and duration on recovery o2. J Appl Physiol 1980;60:169-74.

14. Jakson AS, Pollock ML. Practical assessment of body composition. Physiol Sports Med 1984;13:76-82.

15. Luckaski HC, Bolonchuk, WW, Hall CB; Siders WA. Validation of tetrapolar bioelectrical impedance method to assess human body composition. J Appl Physiol 1986;60:1327-32.

16. McConnell TR. Practical Considerations in the testing of O2max in runners. Sports Med 1988;5:57-68.

17. McArdle WD; Katch FI; Katch VL. Fisiologia do Exercício Energia Nutrição e Desempenho Humano. 5 ed. Rio de Janeiro - RJ. Guanabara; 2001.

18. Flint A, Raben A, Blundell JE, Astrup A. reproducibility, power and validity of visual analogue scales in assessment of appetite sensations in single test meal studies. Int J Obesity Relat Disord 2000;24:38-48.

19. Stubbs RJ, Hughes DA, Johnstone AM, Horgan GW, King N, Blundell JE. A decrease in physical activity affects appetite, energy, and nutrient balance in lean men feeding ad libitum. Am J Clin Nutr 2004;79:62-9.

20. Borsheim E, Bahr R. Effect of exercise intensity, duration and mode on post-exercise oxygen consumption. Sports Med 2003; 14:1037-1060.

21. Markofski MM, Braun WA, Hawthorne WE. Acute EPOC response in women to circuit training and treadmill exercise of matched oxygen consumption. Eur J Appl Physiol 2005;94:500-4.

22. Ohkawara K, Tanaka S, Takata K, Tabata I. Twentyfour-analysis of elevated energy expenditure after physical activity in a metabolic chamber: models of daily total energy expenditure. Am J Clin Nutr 2008; 87:1268-76. 
23. Brooks GA, Fahey TD, White TP, Baldwin KM. Exercise Physiology: Human Bioenergetics and its applications. 3rd edition. Macmillan Publishing Company: New York, 2000.

24. Pillard F, Van Wymelbeke V, Garrigue E, Moro C, Crampes F, Guilland JC, et al. Lipid oxidation in overweight men after exercise and food intake. Metabolism 2010; 59:267-274.

25. King NA, Caudwell PP, Hopkins M, Stubbs JR, Naslund E, Blundell JE. Dual-process action of exercise on appetite control: increase in orexigenic drive but improvement in meal-induced satiety. Am J Clin Nutr 2009; 90: 921-7.

26. Broom DR, Batterham RL, King AJ, Stensel DJ. Influence of resistance and aerobic exercise on hunger, circulating levels of acylated ghrelin, and peptide YY in healthy males. Am J Physiol Regul Integr Comp Physiol 2009; 96:29-35

27. Broom DR, Stensel JD, Bishop NC, Burns SF, and Miyashita M. Exercise-induced suppression of acylated ghrelin in humans. J Appl Phys 2007; 102:2165-2171.

28. Shorten AL, Wallman KE, Guelfi KJ. Acute effect of environmental temperature during exercise on subsequent energy intake in active men. Am J Clin Nutr 2009;90:1215-21.
29. Graaf C, Blom W Smeets P, Stafleu A, Hendriks H. Biomarkers of satiation and satiety. Am J Clin Nutr 2004; 79:946-61.

\section{Endereço para correspondência}

Wagner Luiz do Prado

Escola Superior de Educação Física- (ESEF/UPE)

Campus Universitário

Rua Arnóbio Marques, 310. Santo Amaro

CEP: 50.100.13 - Recife, PE.

E-mail: wagner.prado@upe.br; wlp_personal@yahoo.com.br 